\title{
Wnt3a suppresses Pseudomonas aeruginosa-induced inflammation and promotes bacterial killing in macrophages
}

\author{
KANG CHEN $^{1,2}$, QIANG FU ${ }^{1}$, DANDAN LI ${ }^{3,4}$, YONGJIAN WU ${ }^{3,4}$, SHIJUN SUN ${ }^{1}$ and XIUMING ZHANG ${ }^{1}$ \\ ${ }^{1}$ Division of Clinical Laboratory, Zhongshan Hospital of Sun Yat-Sen University, Zhongshan, Guangdong 528403; \\ ${ }^{2}$ Laboratory Medicine Center, Nangfang Hospital, Southern Medical University, Guangzhou, Guandong 510515; \\ ${ }^{3}$ Department of Immunology, Institute of Human Virology, Zhongshan School of Medicine, Sun Yat-Sen University; \\ ${ }^{4}$ Key Laboratory of Tropical Diseases Control, Ministry of Education, Guangzhou, Guangdong 510080, P.R. China
}

Received February 23, 2015; Accepted January 11, 2016

DOI: $10.3892 / \mathrm{mmr} .2016 .4869$

\begin{abstract}
Pseudomonas aeruginosa (PA) is a common Gram-negative bacterium and can cause serious infections, including hospital-acquired pneumonia, suppurative bacterial keratitis and acute burn wound infection. The pathogenesis of PA infections is closely associated with excessive inflammatory responses and bacterial virulence factors. Wingless-type MMTV integration site family, member 3A (Wnt3a), an upstream mediator in the canonical Wnt signaling pathway, has been implicated as a regulator of inflammation. However, its role in PA-induced inflammation and bacterial clearance remains unknown. In the present study, the efficacy of Wnt3a conditioned media (Wnt3a-CM) was assessed using western blotting and immunofluorescence, which showed that $\beta$-catenin, a downstream molecule of Wnt3a, was upregulated and translocated to the nucleus following exposure to $50 \% \mathrm{Wnt} 3 \mathrm{a}-\mathrm{CM}$ for $6 \mathrm{~h}$. To explore the role of Wnt3a in PA-induced inflammation, the mRNA levels of pro-inflammatory cytokines and apoptosis in macrophages were measured using reverse transcription-quantitative polymerase chain reaction and flow cytometry, respectively. This indicated that Wnt3a suppressed inflammation by reducing the production of pro-inflammatory cytokines and by promoting apoptosis in macrophages. Furthermore, the mechanism of macrophage-mediated bacterial killing was investigated, and the results indicated that Wnt3a enhanced macrophage-mediated intracellular bacterial killing via the induction of the production of cathelicidin-related antimicrobial peptide and $\beta$-defensins 1 . Taken together, the current study explored the role of $\mathrm{Wnt} 3 \mathrm{a}$ in inflammation and bacterial
\end{abstract}

Correspondence to: Mr. Xiuming Zhang, Division of Clinical Laboratory, Zhongshan Hospital of Sun Yat-Sen University, 2 Sunwendong Road, Zhongshan, Guangdong 528403, P.R. China E-mail: zxm0760@163.com

Key words: wingless-type MMTV integration site family, member 3A, inflammation, apoptosis, bacterial killing, antimicrobial peptides invasion, which may provide an improved understanding of host resistance to PA infection.

\section{Introduction}

Pseudomonas aeruginosa (PA), a Gram-negative opportunistic bacterium, is a leading cause of hospital-acquired pneumonia (1) and is responsible for suppurative bacterial keratitis (2) and acute burn wound infection (3). Clinically, antibiotics including fluoroquinolones (4), aminoglycosides (5) and third-generation cephalexin (6) are used to eradicate the bacteria. However, with increasing difficulties in achieving satisfactory efficacy with current antibiotic regimens, efforts have focused on investigating host defense mechanisms against bacterial infections.

The host inflammatory response is a self-protective strategy to control bacterial burden, and is characterized by the accumulation of inflammatory cells and cytokines (7). During infection, inflammatory cells, such as macrophages, are rapidly recruited to kill the invading pathogens (7). Macrophages also produce various pro-inflammatory cytokines including interleukin 6 (IL-6), IL-1 $\beta$, tumor necrosis factor $\alpha$ (TNF- $\alpha$ ) and macrophage inflammatory protein 2 (MIP-2), which function to enhance the antibacterial immune response $(2,7,8)$. These inflammatory mediators promote bacterial clearance, however, if uncontrolled may result in tissue damage (2). Therefore, a combined strategy to control both the bacterial burden and inflammation is critical for the clinical treatment of PA infection.

Wingless-type MMTV integration site family, member 3A (Wnt3a) is a multifunctional molecule that has been reported to have the ability to modulate host inflammation (9-11). As a cysteine-rich secretory glycoprotein, Wnt3a secretes and binds to its respective dimeric cell surface receptors, frizzled proteins and low-density lipoprotein receptor-related protein $5 / 6$, inhibiting the activity of the 'destruction complex' and the subsequent degradation of $\beta$-catenin (12). Stabilized $\beta$-catenin translocates into the nucleus, binds to transcription factors such as $\mathrm{T}$ cell factor and lymphoid-enhancing factor and promotes the expression of target genes that are involved in proliferation, and carcinogenesis (12). 
Although studies have indicated a potential association of Wnts with several inflammatory diseases, including diabetic retinopathy (9), inflammatory bowel disease (10) and rheumatoid arthritis (11), whether Wnt3a serves a positive or negative role in the inflammatory response remains controversial. It has been reported that $\mathrm{Wnt} 3 \mathrm{a}$ increased the secretion of IL- $1 \beta$ and IL-6 in fibrotic alveolar epithelia via activating Wnt/ $\beta$-catenin signaling (13). However, other studies have demonstrated that Wnt family members (such as Wnt2 and Wnt11) suppress bacterial-induced IL-8 secretion in epithelial cells $(14,15)$. To date, little is known regarding the activity of Wnt3a in PA-induced inflammatory responses.

Programmed cell death (apoptosis) is an important strategy to control host inflammatory responses. However, the role of $\mathrm{Wnt} 3 \mathrm{a}$ in regulating apoptosis remains elusive. Zimmerman et al (16) demonstrated that Wnt3a enhanced apoptosis in melanoma cells, while Gui et al (17) indicated that Wnt3a stimulated $\beta$-cell proliferation and inhibited cytokine-induced $\beta$-cell apoptosis. Thus, the role of Wnt3a in regulating $\mathrm{PA}$-induced macrophage apoptosis remains to be determined.

Liu et al (14) reported that Wnt family members have different functions in pathogen clearance. Wnt2 had no effect on inhibiting bacterial invasion (14), whereas Wnt11 significantly reduced Salmonella invasion in epithelial cells (15). In addition, Wnt/ $\beta$-catenin signaling (canonical Wnt signaling) has been implicated in modulating the generation of the major components in the oxygen-dependent microbicidal system, such as reactive oxygen species (ROS) (18) and nitric oxide (NO) (19). Furthermore, studies have demonstrated that antimicrobial peptides (the major components of the oxygen-independent microbicidal system) such as cathelicidin-related antimicrobial peptide (CRAMP) $(20,21)$ and $\beta$-defensin (BD) (22) are required in the host resistance to PA infection, by controlling the host inflammatory response and bacterial burden. However, to date, little is known regarding the activity of $\mathrm{Wnt} 3 \mathrm{a}$ in macrophage-mediated bacterial killing ability.

In the present study, Wnt3a was demonstrated to serve an anti-inflammatory role by suppressing the expression of pro-inflammatory cytokines and promoting macrophage apoptosis. Furthermore, bacterial clearance studies indicated that Wnt3a enhanced intracellular bacterial killing via inducing the expression of CRAMP and BD1.

\section{Materials and methods}

Cell culture.Conditioned media containing Wnt3a(Wnt3a-CM) was prepared from mouse L cells stably expressing Wnt3a (ATCC ${ }^{\circledR}$ CRL-2647 ${ }^{\mathrm{TM}}$; American Type Culture Centre, Manassas, VA, USA), and control conditioned media (Ctl-CM; American Type Culture Centre) was obtained from parental L cells (ATCC ${ }^{\circledR}$ CRL-2648 ${ }^{\mathrm{TM}}$; American Type Culture Centre). RAW264.7 murine macrophage-like cells (ATCC \#TIB-71; American Type Culture Centre) were cultured as described previously (23). Briefly, cells were cultured in Dulbecco's modified Eagle's media (DMEM) supplemented with $10 \%$ (v/v) fetal bovine serum (FBS), $1 \%$ penicillin-streptomycin and $1 \%$ L-glutamine (all purchased from Invitrogen; Thermo Fisher Scientific, Inc., Waltham, MA, USA) at $37^{\circ} \mathrm{C}$ in $5 \% \mathrm{CO}_{2}$. Cells were starved in DMEM containing 2\% FBS overnight and then exposed to Wnt3a-CM or Ctl-CM.

Western blotting. RAW264.7 cells were treated with various concentrations $(10,20,30,40$ or $50 \%)$ of Wnt3a-CM for $6 \mathrm{~h}$ or with $50 \%$ Wnt3a-CM for 6, 12, 24, 36 or 48 h. Subsequently, the cells were washed three times with ice-cold PBS and then treated with lysis buffer containing $1 \mathrm{mM}$ phenylmethylsulfonyl fluoride, $1 \%(\mathrm{v} / \mathrm{v})$ protease inhibitor cocktail and $1 \mathrm{mM}$ dithiothreitol (all purchased from Sigma-Aldrich). Subsequently, the cell lysate was obtained by centrifugation at $12,000 \times \mathrm{g}$ for $15 \mathrm{~min}$ at $4^{\circ} \mathrm{C}$, after which the protein concentration of the supernatant was determined using the Quick Start Bradford protein assay (Bio-Rad Laboratories, Inc., Hercules, CA, USA). $30 \mu \mathrm{g}$ of each sample was separated by $10 \%$ sodium dodecyl sulfate polyacrylamide gel electrophoresis (Beijing Dingguo Changsheng Biotechnology Co., Ltd., Beijing, China), and then transferred to nitrocellulose membranes (Pall Corporation, Port Washington, NY, USA). The membranes were blocked with 5\% non-fat milk in PBS containing 0.1\% Tween-20 and incubated with the primary rabbit monoclonal antibodies against $\beta$-catenin $(1: 1,000$; cat. no. 8480), cleaved-caspase 3 (1:1,000; cat. no. 9664), cleaved-poly ADP ribose polymerase (PARP; 1:1,000; cat. no. 5625) and $\beta$-actin (1:5,000; cat. no. 3700; all Cell Signaling Technology, Inc.) at $4^{\circ} \mathrm{C}$ overnight, followed by incubation with IRDye $800 \mathrm{CW}$ donkey anti-rabbit IgG (H+L) antibodies (1:5,000; DkxRb-003-D800NHSX; LI-COR, Inc., Lincoln, NE, USA) for $1 \mathrm{~h}$ at room temperature. Subsequently, the bands were detected using an Odyssey CLx Infrared Imaging System (LI-COR, Inc.), with an excitation wavelength of $780 \mathrm{~nm}$ and an emission wavelength of $820 \mathrm{~nm}$. Band intensities were quantified using the Image Studio software, version 4.0 (LI-COR, Inc.), according to the manufacturer's protocol.

Immunofluorescent staining. A proportion of RAW264.7 cells were infected with PA at a multiplicity of infection (MOI) of 1.0 for $12 \mathrm{~h}$, after which RAW264.7 cells $\left(4 \times 10^{5}\right.$ cells $\left./ \mathrm{ml}\right)$ with or without PA infection were exposed to $50 \%$ Wnt3a-CM or Ctl-CM for $6 \mathrm{~h}$ and then seeded onto sterile glass cover slips, cultured overnight and fixed in $4 \%$ formaldehyde (Sigma-Aldrich, St. Louis, MO, USA) at $4^{\circ} \mathrm{C}$. Subsequently, the sections were blocked with $5 \%$ bull serum albumin in phosphate-buffered saline (PBS) at room temperature for $1 \mathrm{~h}$, after which they were incubated with rabbit anti-mouse $\beta$-catenin (1:200; cat. no. 8480; Cell Signaling Technology, Inc., Danvers, MA, USA) at $4^{\circ} \mathrm{C}$ overnight. The sections were then incubated with $\mathrm{Cy} 3$-conjugated goat anti-rabbit $\mathrm{IgG}$ (1:1,000; cat. no. AP187C, EMD Millipore, Billerica, MA, USA) at room temperature for $1 \mathrm{~h}$. Finally, sections were incubated with 4,6-diamino-2-phenyl indole (DAPI, 1:10,000; Sigma-Aldrich) at room temperature for $5 \mathrm{~min}$ for nuclear staining. The controls were similarly treated, although the primary antibody was replaced with isotype matched $\mathrm{IgG}$. The sections were visualized using the Olympus BX41 microscope (Olympus Corporation, Tokyo, Japan).

Reverse transcription-quantitative polymerase chain reaction (RT-qPCR). Total RNA was isolated from the RAW264.7 cells using TRIzol ${ }^{\circledR}$ reagent (Invitrogen; Thermo Fisher 
Scientific, Inc.), and the purity of the RNA was determined by measuring the ratio of absorbance at 260 and $280 \mathrm{~nm}$ using the NanoDrop ${ }^{\mathrm{TM}} 2000$ Spectrophotometer (Thermo Fisher Scientific, Inc.). Total RNA ( $1 \mu \mathrm{g})$ was reverse transcribed into cDNA using the High-Capacity RNA-to-cDNA kit (Applied Biosystems; Thermo Fisher Scientific, Inc.), and then amplified using the SYBR Green Master Mix (Bio-Rad Laboratories, Inc.), according to the manufacturer's protocols. The primer sequences for IL-6, IL-1 $\beta$, MIP-2, TNF- $\alpha$, mCRAMP, mBD1 and $\beta$-actin are listed in Table I. RT-qPCR reactions were performed using the CFX96 Real-Time PCR System (Bio-Rad Laboratories, Inc.) with the following cycling conditions: $95^{\circ} \mathrm{C}$ for $30 \mathrm{sec}$, followed by 40 cycles of $95^{\circ} \mathrm{C}$ for $5 \mathrm{sec}$ and $60^{\circ} \mathrm{C}$ for $10 \mathrm{sec}$. Relative mRNA expression levels were calculated following normalization to $\beta$-actin using the $2^{-\Delta \Delta \mathrm{Cq}}$ method and the Prism 5.0 software (GraphPad Software, Inc., La Jolla, CA, USA).

Flow cytometric analysis of apoptosis. A proportion of RAW264.7 cells were infected with PA at a MOI of 0.5 for $24 \mathrm{~h}$. The apoptosis of RAW264.7 cells with or without PA infection was assessed by flow cytometry using a fluorescein isothiocyanate (FITC)-annexin V apoptosis detection kit (BD Biosciences, Inc., Franklin Lakes, NJ, USA) according to the manufacturer's protocol. Briefly, the cells $\left(5 \times 10^{5}\right)$ were pooled, washed and resuspended in $500 \mu \mathrm{l}$ binding buffer, followed by the addition of $5 \mu \mathrm{l}$ annexin V-FITC and $5 \mu \mathrm{l}$ propidium iodide (PI). Subsequently, the cells were incubated at room temperature in the dark for $15 \mathrm{~min}$, and analyzed by flow cytometry (Beckman Coulter EPICS XL/MCL; Beckman Coulter, Brea, CA, USA), with an excitation wavelength of $488 \mathrm{~nm}$ and an emission wavelength of 530 for FITC (green) and 575-610 nm for PI (orange). Viable cells were unstained by annexin V or PI, early apoptotic cells were stained by annexin $\mathrm{V}$ only, and late apoptotic cells were stained by annexin V and PI.

Intracellular bacterial killing assay. RAW264.7 cells were cultured in a 6-well plate and then exposed to $50 \%$ Wnt3a-CM or Ctl-CM for $6 \mathrm{~h}$, followed by PA challenge at a MOI of 25 for 1 and $2 \mathrm{~h}$, respectively. Subsequently, the cells in one well were treated with $300 \mu \mathrm{g} / \mathrm{ml}$ gentamicin (Sigma-Aldrich) for $30 \mathrm{~min}$ in order to kill the extracellular bacteria, and then washed with PBS three times and lysed with $0.1 \%$ Triton-X. Cells in the duplicate well were incubated for a further $1 \mathrm{~h}$ and then lysed in the same way. Serial 10-fold dilutions of each sample were plated on Pseudomonas isolation agar (BD Biosciences, Inc.) in triplicate and incubated overnight at $37^{\circ} \mathrm{C}$. For intracellular bacterial killing, the efficiency was calculated using the following equation: Intracellular bacterial killing $=$ [colony forming units $(\mathrm{CFU})(1 \mathrm{~h})-\mathrm{CFU}$ (2 h)]/CFU (1 h) x 100\%.

Phagocytosis assay. Phagocytosis was assayed by flow cytometry as described previously (24). Briefly, PA was incubated with Filmtracer Green Biofilm (FTGB, 1:50; Invitrogen; Thermo Fisher Scientific, Inc.) at room temperature for $30 \mathrm{~min}$ in the dark, and then rinsed gently with sterilized water. Subsequently, RAW264.7 cells were exposed to $50 \% \mathrm{Wnt} 3 \mathrm{a}-\mathrm{CM}$ or $\mathrm{Ctl}-\mathrm{CM}$ for $6 \mathrm{~h}$, and then infected with FTGB-stained PA at a MOI of 25. Following
Table I. Nucleotide sequence of the primers used in PCR amplification.

\begin{tabular}{ll} 
Gene & \multicolumn{1}{c}{ Primer sequence $\left(5^{\prime}-3^{\prime}\right)$} \\
\hline$\beta$-actin & F: GATTACTGCTCTGGCTCCTAGC \\
& R: GACTCATCGTACTCCTGCTTGC \\
IL-6 & F: CACAAGTCCGGAGAGGAGAC \\
& R: CAGAATTGCCATTGCACAAC \\
IL-1 $\beta$ & F: CGCAGCAGCACATCAACAAGAGC \\
& R: TGTCCTCATCCTGGAAGGTCCACG \\
MIP-2 & F: TGTCAATGCCTGAAGACCCTGCC \\
& R: AACTTTTGACCGCCCTTGAGAGTGG \\
TNF- $\alpha$ & F: CACAGAAAGCATGATCCGCGAC \\
& R: TGCCACAAGCAGGAATGAGAAGAG \\
mCRAMP & F: AGCTACAGGGATGCTGTGCT \\
& R: TCACTCGGAACCTCAGACT \\
mBD1 & F: GGCATTCTCACAAGTCTTGGACGAAG \\
& R:AGCTCTTACAACAGTTGGGCTTATCTGG
\end{tabular}

F, forward; R, reverse; IL, interleukin; MIP, macrophage inflammatory protein; TNF, tumor necrosis factor; mCRAMP, mouse cathelicidin-related antimicrobial peptide; $\mathrm{mBD} 1$, mouse $\beta$-defensin 1 .

$1 \mathrm{~h}$ of incubation, the cells were washed three times with ice-cold PBS to remove nonadherent bacteria. Extracellular fluorescence was quenched by the addition of $0.1 \%$ trypan blue (Sigma-Aldrich) in PBS for $15 \mathrm{~min}$. Cells were collected and analyzed using a Beckman Coulter EPICS XL/MCL instrument, with an excitation wavelength of $488 \mathrm{~nm}$ and an emission wavelength of $514 \mathrm{~nm}$.

ROS measurement by flow cytometry. RAW264.7 cells were exposed to Wnt3a-CM or Ctl-CM for $6 \mathrm{~h}$. Following PA challenge, the cells were incubated with a ROS-sensitive probe, 2',7'-dichlorofluorecscin diacetate $\left(\mathrm{H}_{2} \mathrm{DCFDA}\right.$; Invitrogen; Thermo Fisher Scientific, Inc.), at a final concentration of $10 \mathrm{~mm}$. Subsequently, the cells were collected and analyzed using a Beckman Coulter EPICS XL/MCL flow cytometer, with an excitation wavelength of $488 \mathrm{~nm}$ and an emission wavelength of $530 \mathrm{~nm}$. ROS levels were determined by the fluorescence of dichlorofluorecscin (DCF), the deacetylated and oxidized product of $\mathrm{H}_{2}$ DCFDA.

Griess assay. RAW264.7 cells were exposed to Wnt3a-CM or Ctl-CM, and the supernatant from each sample was collected at 12 and $24 \mathrm{~h}$ following PA challenge by centrifugation at $1,000 \mathrm{x} \mathrm{g}$ for $10 \mathrm{~min}$ at $4^{\circ} \mathrm{C}$. NO levels were determined by measuring the levels of the stable end product, nitrite, using the Griess reagent assay (Sigma-Aldrich), according to the manufacturer's protocol. Briefly, $50 \mu \mathrm{l}$ culture supernatant mixed with $50 \mu \mathrm{l}$ sulfanilamide solution was added to wells in duplicate, and incubated for $10 \mathrm{~min}$ at room temperature in the dark. Subsequently, $50 \mu 1 \mathrm{~N}$-1-napthylethylenediamine dihydrochloride solution was added to the wells and incubated for $10 \mathrm{~min}$ at room temperature in the dark. Absorbance at $540 \mathrm{~nm}$ was measured using the iMarkTM Microplate Absorbance Reader (Bio-Rad Laboratories, Inc.). 
A

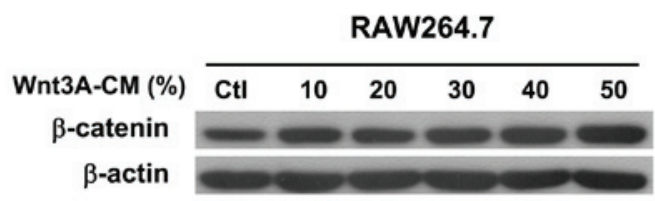

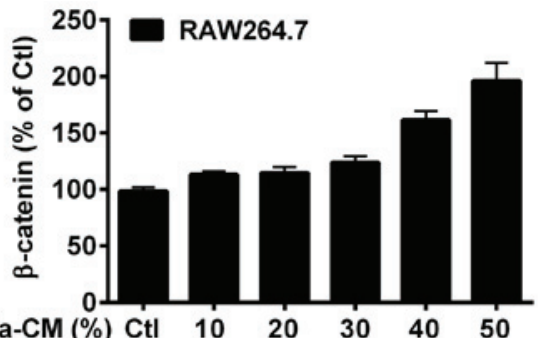

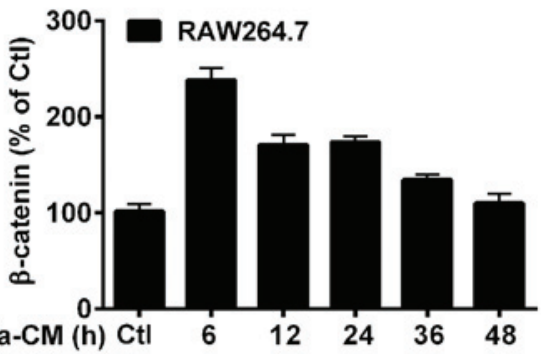

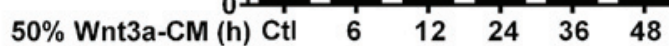

C

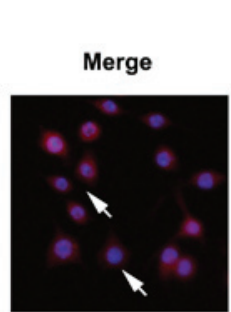

CtI-CM

Cy3
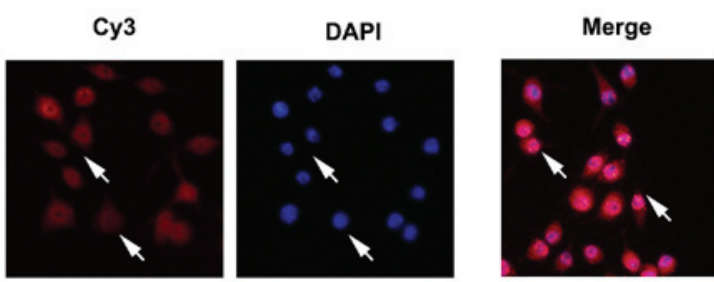

Wnt3A-CM
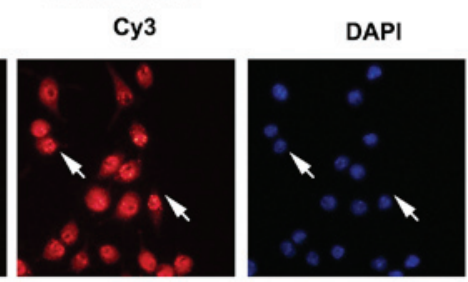

Figure 1. Efficacy of Wnt3a-CM in macrophages. The protein levels of $\beta$-catenin in RAW264.7 cells were measured by western blotting following (A) treatment with Wnt3a-CM at different concentrations and (B) treatment with $50 \%$ Wnt3a-CM for various durations. $\beta$-catenin protein levels were quantitated by calculating relative integrated density values following normalization to $\beta$-actin and presented as the percentage of $\beta$-catenin expression levels in the control treated cells. Data are presented as the mean \pm standard error of the mean for triplicate experiments. (C) Immunofluorescent staining was used to detect $\beta$-catenin expression (red staining) in Wnt3a-CM and Ctl-CM treated RAW264.7 cells. Wnt3a, wingless-type MMTV integration site family, member 3A; Wnt3a-CM, Wnt3a conditioned media; Ctl-CM, control conditioned media; DAPI, 4',6-diamidino-2-phenylindole.

Statistical analysis. Data are presented as the mean \pm standard error of the mean of three independent experiments. Statistical analyses were conducted using the Prism 5.0 software (GraphPad Software, Inc., La Jolla, CA, USA). An unpaired, two-tailed Student's t-test was used to analyze differences between the Wnt3a-CM and Ctl-CM treated groups at the same time point following PA infection. $\mathrm{P}<0.05$ was considered to indicate a statistically significant difference.

\section{Results}

Efficacy of Wnt3a-CM exposure in macrophages. The efficacy of Wnt3a-CM exposure was demonstrated by the expression and distribution of $\beta$-catenin, a central molecule of the canonical Wnt signaling pathway (12). RAW264.7 cells were exposed to Wnt3a-CM at a range of concentrations (Fig. 1A) and to $50 \% \mathrm{Wnt} 3 \mathrm{a}-\mathrm{CM}$ for various durations (Fig. 1B). Western blotting data showed that the protein levels of $\beta$-catenin were markedly upregulated in RAW264.7 cells following exposure to $50 \%$ Wnt3a-CM (Fig. 1A) and for $6 \mathrm{~h}$ (Fig. 1B). Furthermore, immunofluorescence data (Fig. 1C) indicated that $\beta$-catenin (red staining, Cy3-labeled) was upregulated in the cytoplasm and was translocated to the nucleus (DAPI nuclear staining) following exposure to $50 \% \mathrm{Wnt} 3 \mathrm{a}-\mathrm{CM}$ for $6 \mathrm{~h}$.
Wnt3a inhibits pro-inflammatory cytokine secretion in macrophages. To explore the inflammatory regulation of Wnt3a, the expression of pro-inflammatory cytokines was measured by RT-qPCR in Wnt3a-CM and Ctl-CM treated RAW264.7 cells before and after PA infection. The RT-qPCR data showed that Wnt3a suppressed the mRNA levels of IL-6 $(\mathrm{P}<0.05$; Fig. 2A), IL-1 $\beta$ ( $\mathrm{P}<0.01$; Fig. 2B), MIP-2 ( $\mathrm{P}<0.01$; Fig. 2C) and TNF- $\alpha(\mathrm{P}<0.05$; Fig. 2D) following PA infection. In addition, no alteration in pro-inflammatory cytokine expression was detected in Wnt3a-CM and Ctl-CM treated groups prior to PA challenge.

Wnt3a promotes macrophage apoptosis following PA challenge. The role of Wnt3a in modulating macrophage apoptosis was further examined by annexin-V/PI double staining and flow cytometry. The flow cytometry data showed that Wnt3a increased the number of annexin $\mathrm{V}$ positive cells following PA challenge (PA+; Fig. 3A). However, Wnt3a slightly reduced cell apoptosis in the absence of PA infection (PA-; Fig. 3A), which was consistent with the role of Wnt3a in cell proliferation. Additional evidence for the occurrence of apoptosis was obtained by western blot analysis, which measured cleaved caspase 3 and cleaved-PARP, two hallmarks of apoptosis. The western blotting data showed that Wnt3a induced caspase 3 and PARP cleavage in macrophages at 12 and $24 \mathrm{~h}$ 
A

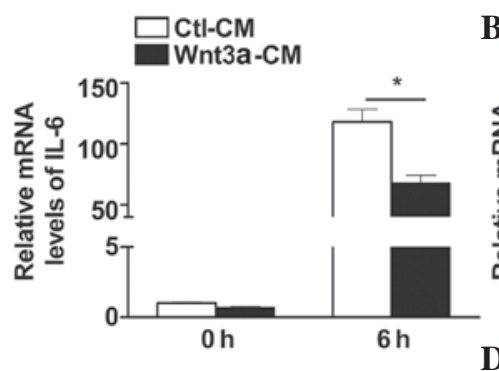

C

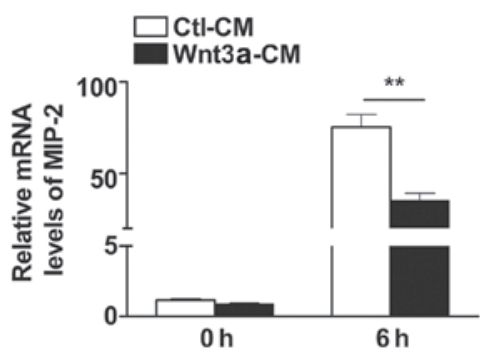

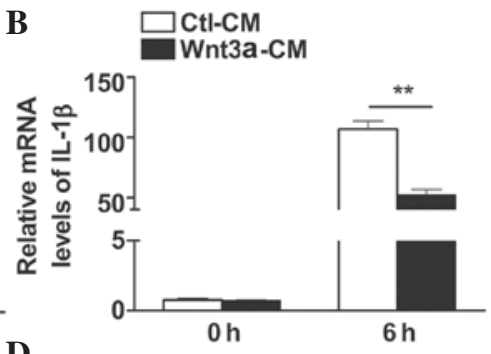

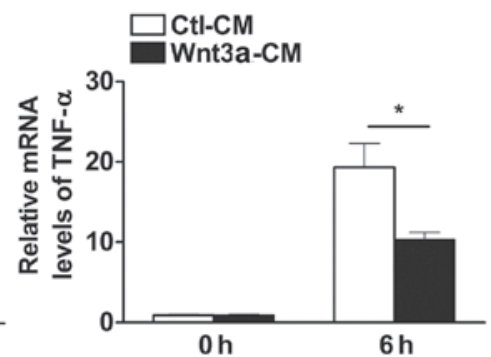

Figure 2. Wnt3a inhibited the expression of pro-inflammatory cytokines in macrophages following infection. The mRNA levels of (A) IL-6, (B) IL-1 $\beta$, (C) MIP-2 and (D) TNF- $\alpha$ were examined by reverse transcription-quantitative polymerase chain reaction in 50\% Wnt3a-CM and Ctl-CM treated RAW264.7 cells with $(6 \mathrm{~h})$ and without $(0 \mathrm{~h}) \mathrm{PA}$ infection. Data are presented as the mean \pm standard error and represent three individual experiments. ${ }^{*} \mathrm{P}<0.05$; ${ }^{* *} \mathrm{P}<0.01$. IL, interleukin; MIP-2, macrophage inflammatory protein 2; TNF- $\alpha$, tumor necrosis factor- $\alpha$; Wnt3a, wingless-type MMTV integration site family, member 3A; Wnt3a-CM, Wnt3a conditioned media; Ctl-CM, control conditioned media; PA, Pseudomonas aeruginosa.

A

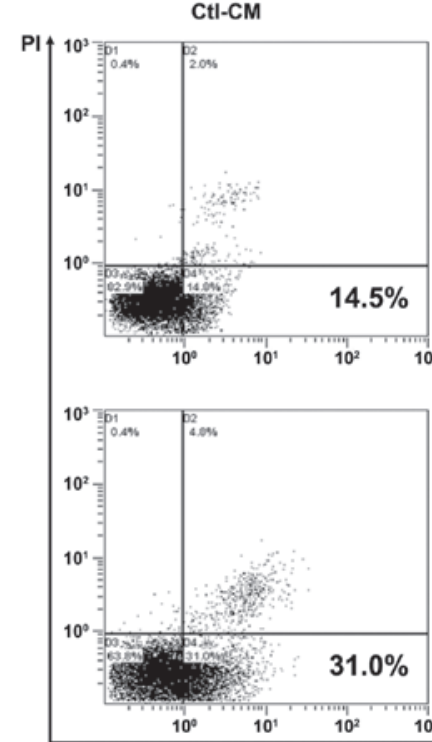

Wnt3a-CM
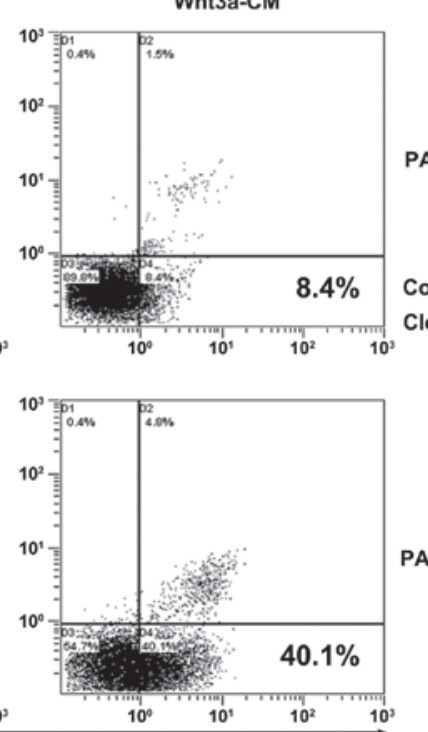

B

PA-

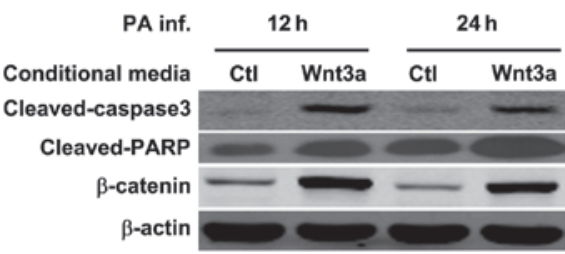

PA+

Figure 3. Wnt3a promoted apoptosis in macrophages following PA infection. (A) Apoptosis in Wnt3a-CM and Ctl-CM treated RAW264.7 cells before PA infection and after PA infection was analyzed by annexin-V/PI double staining and flow cytometry. (B) Western blotting was used to measure the protein levels of cleaved caspase 3, cleaved PARP and $\beta$-catenin in 50\% Wnt3a-CM and Ctl-CM treated RAW264.7 cells at 12 and $24 \mathrm{~h}$ following PA infection. Data represent three individual experiments. PA, Pseudomonas aeruginosa; Wnt3a, wingless-type MMTV integration site family, member 3A; Wnt3a-CM, Wnt3a conditioned media; Ctl-CM, control conditioned media; PA-, without PA infection; PA+, with PA infection; PARP, poly ADP-ribose polymerase.

following PA stimulation (Fig. 3B), compared with the Ctl-CM treated cells. Furthermore, the efficacy of Wnt3a-CM treatment in RAW264.7 cells (Fig. 3B) was confirmed by western blotting, as indicated by the upregulation of $\beta$-catenin.

Wnt3a enhances intracellular bacterial killing however, not phagocytosis following PA infection. It has been previously reported that Wnt11 prevents bacterial invasiveness in intestinal epithelial cells (15), therefore, the present study investigated whether Wnt3a modulates the process of bacterial clearance.
Bacterial clearance was assessed by using a bacterial killing assay based on plate counts (Fig. 4A) and a phagocytosis assay using flow cytometry (Fig. 4B). Bacterial killing data indicated that Wnt3a promoted bacterial killing following PA challenge in RAW264.7 cells ( $\mathrm{P}<0.05$; Fig. 4A), and the phagocytosis assay indicated alterations in the uptake of PA in Wnt3a-CM and Ctl-CM treated RAW264.7 cells (Fig. 4B). These data demonstrated that Wnt3a enhances macrophage-mediated intracellular killing of PA, however, was not involved in the process of phagocytosis. 
A

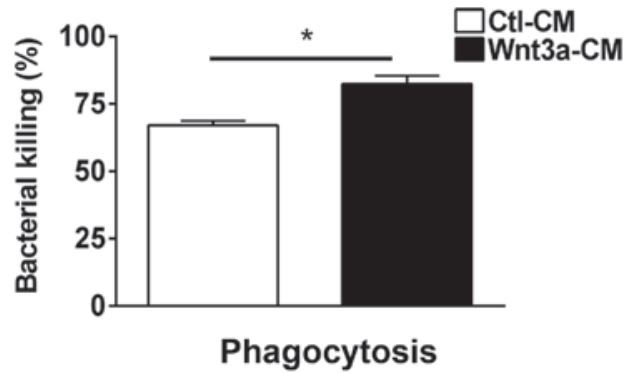

B

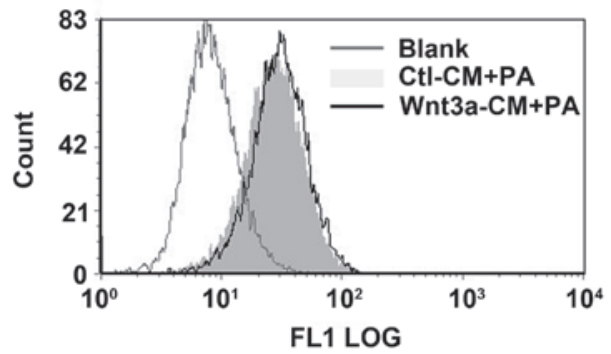

Figure 4. Wnt3a promoted intracellular bacterial killing but not phagocytosis in Pseudomonas aeruginosa-challenged macrophages. (A) Bacterial killing was measured using plate counting in 50\% Wnt3a-CM and Ctl-CM treated RAW264.7 cells. Data are presented as the mean \pm standard error of the mean for triplicate experiments. (B) Phagocytosis was measured using flow cytometry in 50\% Wnt3a-CM and Ctl-CM treated RAW264.7 cells. Data represent three individual experiments. ${ }^{*} \mathrm{P}<0.05$. Wnt3a, wingless-type MMTV integration site family, member 3A; Wnt3a-CM, Wnt3a conditioned media; $\mathrm{Ctl}-\mathrm{CM}$, control conditioned media.

Wnt3a promotes macrophage-mediated bacterial killing by elevating CRAMP and BDI levels, however, not ROS or NO production. To explore the microbicidal mechanisms involved during PA infection, oxygen-dependent (ROS and NO) and oxygen-independent microbicidal systems (antimicrobial peptides) were measured in Wnt3a-CM and Ctl-CM treated RAW264.7 cells following PA challenge. No alterations between the two groups were observed in PA-induced ROS production, as indicated by the percentage of DCF-positive cells (Fig. 5A), and NO levels, as indicated by the measurement of the stable end product nitrate (Fig. 5B). However, the results showed that the mRNA levels of CRAMP $(\mathrm{P}<0.05$; Fig. 5C) and $\mathrm{BD} 1(\mathrm{P}<0.05$; Fig. 5D) were significantly upregulated in Wnt3a-CM and Ctl-CM treated RAW264.7 cells following PA infection. These results demonstrated that Wnt3a enhances macrophage-mediated intracellular killing via the antimicrobial peptides CRAMP and BD1, but not by ROS and NO production.

\section{Discussion}

As an upstream mediator in the Wnt/ $\beta$-catenin pathway, Wnt3a serves a critical role in modulating host inflammation (9-11). However, the function of Wnt3a in regulating PA-induced host inflammation and the associated microbicidal mechanisms remain unclear. The present study demonstrated that Wnt3a suppressed inflammation by reducing the expression of pro-inflammatory cytokines and promoting apoptosis in macrophages. In addition, it was observed that Wnt3a promoted macrophage-mediated intracellular bacterial killing by elevating the levels of the antimicrobial peptides CRAMP and BD1. Taken together, these results shed light on the regulation of Wnt3a in bacterial infectious diseases.
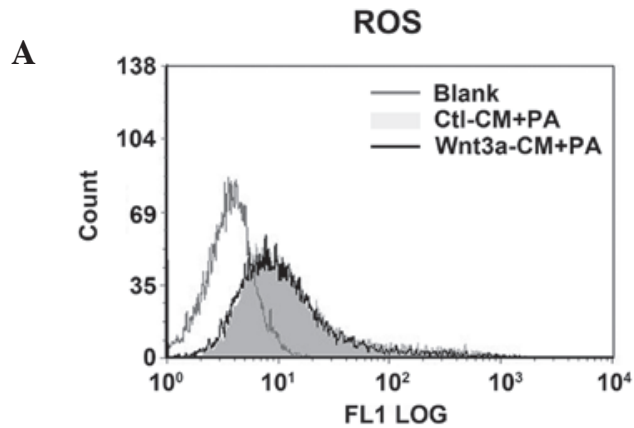

B

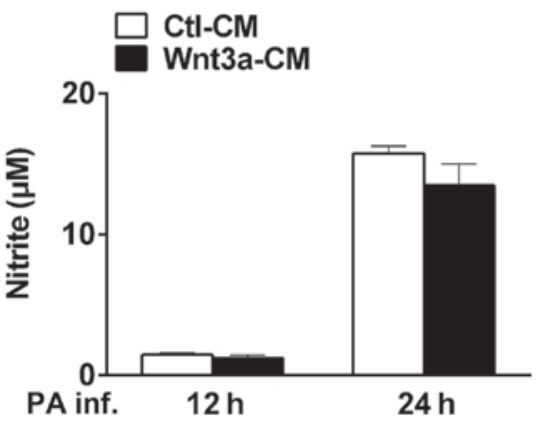

C
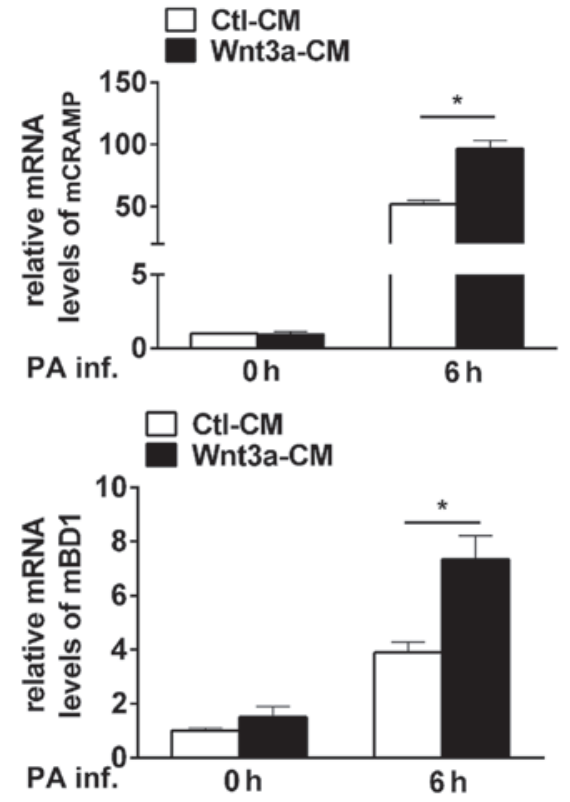

Figure 5. Wnt3a promoted macrophage-mediated bacterial killing by elevating CRAMP and BD1 levels but not ROS or NO production. (A) ROS production was measured using flow cytometry in $50 \% \mathrm{Wnt} 3 \mathrm{a}-\mathrm{CM}$ and Ctl-CM treated RAW264.7 cells following PA infection. (B) NO levels were determined by measuring the stable end product nitrite in $50 \%$ Wnt3a-CM and Ctl-CM treated RAW264.7 cells at 12 and $24 \mathrm{~h}$ after PA infection. The mRNA levels of (C) mCRAMP and (D) mBD1 were measured in 50\% Wnt3a-CM and Ctl-CM treated RAW264.7 cells without $(0 \mathrm{~h})$ and with $(6 \mathrm{~h})$ $\mathrm{PA}$ infection. Data are presented as the mean \pm standard error of the mean of triplicate experiments. ${ }^{*} \mathrm{P}<0.05$. (m)CRAMP, (mouse) cathelicidin-related antimicrobial peptide; (m)BD1, (mouse) $\beta$-defensin 1 ; ROS, reactive oxygen species; Wnt3a, wingless-type MMTV integration site family, member 3A; Wnt3a-CM, Wnt3a conditioned media; Ctl-CM, control conditioned media; PA, Pseudomonas aeruginosa.

Previous studies have demonstrated that Wnt3a serves a pro-inflammatory role, by enhancing the expression of IL-1 $\beta$ and IL-6 in fibrotic alveolar epithelia (13). However, other studies have reported that Wnt family members (Wnt2 and Wnt11) inhibited Salmonella-induced IL-8 
expression in intestinal epithelia cells $(14,15)$. Furthermore, Neumann et al (25) reported that Wnt3a reduced TNF release in Mycobacterium tuberculosis-infected macrophages (25). These studies suggest that Wnt3a may exert anti-inflammatory effects in bacterial-induced inflammatory responses. However, the inflammatory cytokine profiles orchestrated by bacteria stimulation may vary, depending on the cell type and species of bacteria. The current study indicated that Wnt3a served an anti-inflammatory role by reducing the expression of pro-inflammatory cytokines including IL- $1 \beta$, IL-6, MIP-2 and TNF- $\alpha$ in PA-challenged macrophages. The role of Wnt3a in regulating pro-inflammatory cytokines is consistent with a previous study that demonstrated that $\beta$-catenin inhibited the expression of pro-inflammatory cytokines in PA-infected macrophages (23), which suggests that Wnt3a may regulate PA-induced inflammatory cytokines via its downstream mediator $\beta$-catenin. A potential mechanism underlying the inflammatory regulation of Wnt3a may be that Wnt binding stabilizes the transcription factor $\beta$-catenin, which in turn enters the nucleus and negatively regulates nuclear factor- $\kappa \mathrm{B}$, leading to the suppression of the expression of pro-inflammatory cytokines $(26,27)$.

Apoptosis is another strategy employed by the host to control excessive inflammatory responses. Previous studies have demonstrated that Wnt3a promotes the apoptosis of melanoma cells (16) and Bacillus Calmette-Guerin-infected RAW264.7 cells (28); however, it exhibited anti-apoptotic activity in $\beta$-cells (17) and increased the proliferation of heart valve interstitial cells (29). The present study demonstrated that Wnt3a enhanced PA-induced apoptosis in macrophages, which is indispensable for the control of robust inflammatory responses, contributing to the role of Wnt3a in bacterial-induced anti-inflammatory effects.

Inflammatory mediators are critical for the host defense, and lead to clearance of the infectious pathogen (7). However, in the present study, Wnt3a was observed to promote bacterial killing, which appears to be in conflict with its anti-inflammatory activity. Therefore, additional bactericidal mechanisms that may be involved in the process of macrophage-mediated bacterial killing triggered by Wnt3a were investigated. Phagocytic clearance by macrophages is key to the endogenous control of PA (1). However, the present study indicated that Wnt3a had no influence on phagocytosis. It has been reported that $\mathrm{Wnt} 3 / \beta$-catenin signaling promotes ROS (18) and NO (19) production, with these being major components in the oxygen-dependent microbicidal system. However, the present study observed no alteration in the generation of NO or ROS in Wnt3a-CM and Ctl-CM treated macrophages following PA infection. In addition to the oxygen-dependent pathway (e.g., NO/ROS), immune cells possess an oxygen-independent pathway (e.g., antimicrobial peptide, lysozymes or lysosomal hydrolytic enzymes) to fight against invading microorganisms $(30,31)$. It has been previously reported that CRAMP-deficient B6 mice are more susceptible to PA ocular infection than wild-type mice (20). Hazlett and $\mathrm{Wu}$ (22) demonstrated that defensins, as a major family of antimicrobial peptides, exert widely antimicrobial activity (22). The present study observed that Wnt3a promoted CRAMP and BD1 expression, indicating that the antimicrobial activity of Wnt3a may be associated with the induction of CRAMP and BD1.
Inflammation and bacterial virulence contribute to infectious diseases; however, treating bacterial infection with antibiotics does not commonly prevent pathology caused by an excessive immune response. The present study demonstrated that Wnt3a suppressed inflammation and enhanced bacterial killing, thus suggesting that Wnt3a may be considered a therapeutic strategy for the treatment of PA infection.

\section{Acknowledgements}

The present study was supported by grants from the National Natural Science Foundation of China (grant no. 81401645) and Guangdong Medical Science Foundation (grant no. B2014447).

\section{References}

1. Lovewell RR, Patankar YR and Berwin B: Mechanisms of phagocytosis and host clearance of Pseudomonas aeruginosa. Am J Physiol Lung Cell Mol Physiol 306: L591-L603, 2014.

2. Hazlett LD: Corneal response to Pseudomonas aeruginosa infection. Prog Retin Eye Res 23: 1-30, 2004.

3. Bielecki P, Glik J, Kawecki M and Martins dos Santos VA: Towards understanding Pseudomonas aeruginosa burn wound infections by profiling gene expression. Biotechnol Lett 30: 777-790, 2008.

4. Kowalski RP, Romanowski EG, Mah FS, Shanks RM and Gordon YJ: Topical levofloxacin $1.5 \%$ overcomes in vitro resistance in rabbit keratitis models. Acta Ophthalmol 88: e120-e125, 2010

5. McCormick C, Caballero A, Tang A, Balzli C, Song J and O'Callaghan R: Effectiveness of a new tobramycin $(0.3 \%)$ and dexamethasone $(0.05 \%)$ formulation in the treatment of experimental Pseudomonas keratitis. Curr Med Res Opin 24: 1569-1575, 2008.

6. Mohammadpour M, Mohajernezhadfard Z, Khodabande A and Vahedi P: Antibiotic susceptibility patterns of Pseudomonas corneal ulcers in contact lens wearers. Middle East Afr J Ophthalmol 18: 228-231, 2011.

7. Hazlett LD: Pathogenic mechanisms of P. aeruginosa keratitis: A review of the role of T cells, Langerhans cells, PMN and cytokines. DNA Cell Biol 21: 383-390, 2002.

8. Kernacki KA, Goebel DJ, Poosch MS and Hazlett LD: Early cytokine and chemokine gene expression during Pseudomonas aeruginosa corneal infection in mice. Infect Immun 66: 376-379, 1998.

9. Zhou T, Hu Y, Chen Y, Zhou KK, Zhang B, Gao G and Ma JX: The pathogenic role of the canonical Wnt pathway in age-related macular degeneration. Invest Ophthalmol Vis Sci 51: 4371-4379, 2010.

10. You J, Nguyen AV, Albers CG, Lin F and Holcombe RF: Wnt pathway-related gene expression in inflammatory bowel disease. Dig Dis Sci 53: 1013-1019, 2008.

11. Miao CG, Yang YY, He X, Li XF, Huang C, Huang Y, Zhang L, Lv XW, Jin Y and Li J: Wnt signaling pathway in rheumatoid arthritis, with special emphasis on the different roles in synovial inflammation and bone remodeling. Cell Signal 25: 2069-2078, 2013.

12. Wodarz A and Nusse R: Mechanisms of Wnt signaling in development. Annu Rev Cell Dev Biol 14: 59-88, 1998.

13. Aumiller V, Balsara N, Wilhelm J, Günther A and Königshoff M: WNT/ $\beta$-catenin signaling induces IL- $1 \beta$ expression by alveolar epithelial cells in pulmonary fibrosis. Am J Respir Cell Mol Biol 49: 96-104, 2013.

14. Liu X, Lu R, Wu S, Zhang YG, Xia Y, Sartor RB and Sun J: Wnt2 inhibits enteric bacterial-induced inflammation in intestinal epithelial cells. Inflamm Bowel Dis 18: 418-429, 2012.

15. Liu X, Wu S, Xia Y, Li XE, Xia Y, Zhou ZD and Sun J: Wingless homolog Wnt11 suppresses bacterial invasion and inflammation in intestinal epithelial cells. Am J Physiol Gastrointest Liver Physiol 301: G992-G1003, 2011.

16. Zimmerman ZF, Kulikauskas RM, Bomsztyk K, Moon RT and Chien AJ: Activation of Wnt/ $\beta$-catenin signaling increases apoptosis in melanoma cells treated with trail. PLoS One 8: e69593, 2013. 
17. Gui S, Yuan G, Wang L, Zhou L, Xue Y, Yu Y, Zhang J, Zhang $M$, Yang $Y$ and Wang DW: Wnt3a regulates proliferation, apoptosis and function of pancreatic NIT-1 beta cells via activation of IRS2/PI3K signaling. J Cell Biochem 114: 1488-1497, 2013.

18. Kim JS, Yeo S, Shin DG, Bae YS, Lee JJ, Chin BR, Lee CH and Baek SH: Glycogen synthase kinase 3beta and beta-catenin pathway is involved in toll-like receptor 4-mediated NADPH oxidase 1 expression in macrophages. FEBS J 277: 2830-2837, 2010.

19. Du Q, Park KS, Guo Z, He P, Nagashima M, Shao L, Sahai R, Geller DA and Hussain SP: Regulation of human nitric oxide synthase 2 expression by Wnt beta-catenin signaling. Cancer Res 66: 7024-7031, 2006.

20. Huang LC, Reins RY, Gallo RL and McDermott AM: Cathelicidin-deficient (Cnlp -/-) mice show increased susceptibility to Pseudomonas aeruginosa keratitis. Invest Ophthalmol Vis Sci 48: 4498-4508, 2007.

21. Kumar A, Hazlett LD and Yu FS: Flagellin suppresses the inflammatory response and enhances bacterial clearance in a murine model of Pseudomonas aeruginosa keratitis. Infect Immun 76: 89-96, 2008.

22. Hazlett $\mathrm{L}$ and $\mathrm{Wu} \mathrm{M}$ : Defensins in innate immunity. Cell Tissue Res 343: 175-188, 2011

23. Chen K, Yin L, Nie X, Deng Q, Wu Y, Zhu M, Li D, Li M, Wu $\mathrm{M}$ and Huang $\mathrm{X}$ : $\beta$-Catenin promotes host resistance against Pseudomonas aeruginosa keratitis. J Infect 67: 584-594, 2013.
24. Mariencheck WI, Savov J, Dong Q, Tino MJ and Wright JR: Surfactant protein A enhances alveolar macrophage phagocytosis of a live, mucoid strain of $P$. aeruginosa. Am J Physiol 277: L777-L786, 1999

25. Neumann J, Schaale K, Farhat K, Endermann T, Ulmer AJ, Ehlers S and Reiling N: Frizzled1 is a marker of inflammatory macrophages and its ligand Wnt3a is involved in reprogramming Mycobacterium tuberculosis-infected macrophages. FASEB J 24: 4599-4612, 2010.

26. Sun J, Hobert ME, Rao AS, Neish AS and Madara JL: Bacterial activation of beta-catenin signaling in human epithelia. Am J Physiol Gastrointest Liver Physiol 287: G220-G227, 2004.

27. Sun J, Hobert ME, Duan Y, Rao AS, He TC, Chang EB and Madara JL: Crosstalk between NF-kappaB and beta-catenin pathways in bacterial-colonized intestinal epithelial cells. Am J Physiol Gastrointest Liver Physiol 289: G129-G137, 2005.

28. Wu X, Deng G, Hao X, Li Y, Zeng J, Ma C, He Y, Liu X and Wang Y: A caspase-dependent pathway is involved in Wnt/ $\beta$-catenin signaling promoted apoptosis in Bacillus Calmette-Guerin infected RAW264.7 macrophages. Int J Mol Sci 15: 5045-5062, 2014.

29. Xu S and Gotlieb AI: Wnt3a//-catenin increases proliferation in heart valve interstitial cells. Cardiovasc Pathol 22: 156-166, 2013.

30. Thomas EL, Lehrer RI and Rest RF: Human neutrophil antimicrobial activity. Rev Infect Dis 10 (Suppl 2): S450-S456, 1988.

31. Wiesner J and Vilcinskas A: Antimicrobial peptides: The ancient arm of the human immune system. Virulence 1: 440-464, 2010. 